\title{
CREATIVIDAD EN LAS PRÁCTICAS DOCENTES DEL NIVEL INICIAL. UN ESTUDIO DESDE LA EDUCACIÓN MUSICAL
}

\author{
CRIATIVIDADE NAS PRÁTICAS DE ENSINO DO NÍVEL INICIAL. UM \\ ESTUDO DA EDUCAÇÃO MUSICAL
}

Zilli, Lía Rosa

Instituto Superior de Música (UNL)

\section{Resumen}

El presente artículo, tiene como objetivo comunicar los principales resultados a los que se arribó desde un estudio de caso sobre estrategias creativas de enseñanza musical, investigadas en tres profesores de música de la Educación Inicial de la ciudad de Santa Fe en el año 2016 - 2017. Las estrategias creativas de enseñanza musical se relacionaron con el diseño de Secuencias Didácticas Flexibles y fueron consideradas desde los aportes Educación Experiencial en el currículum de la Educación Inicial. De esta manera, se expresa la importancia de pensar, estudiar, diseñar, y evaluar prácticas pedagógico - musicales remitidas a un modelo educativo particular, que logran desde la creatividad del maestro, experiencias de aprendizaje creativo. Se postula en dicho estudio el desarrollo de estrategias creativas de enseñanza musical que parten del juego como mediador, de las experiencias sensoriales como aproximación a la experiencia estética, de la indagación sonora y la construcción a partir del error del alumno como reflexión y evaluación sobre la acción; interpelando de una manera particular las prácticas de enseñanza musical.

Palabras claves: Creatividad. Educación Musical. Educación Inicial. Prácticas docentes. Estrategias creativas de enseñanza. 


\section{CREATIVIDAD EN LAS PRÁCTICAS DOCENTES DEL NIVEL INICIAL. UN ESTUDIO DESDE LA EDUCACIÓN MUSICAL}

\section{Resumo}

O objetivo deste artigo é comunicar os principais resultados que foram obtidos a partir de um estudo de caso sobre estratégias criativas de educação musical, investigadas em três professores de música da Educação Infantil da cidade de Santa Fé no ano de 2016 - 2017 .

As estratégias criativas de educação musical foram relacionadas ao desenho de Seqüências Didáticas Flexíveis e foram consideradas a partir das contribuições da Educação Experiencial no currículo da Educação Básica. Desse modo, expressa - se a importância de pensar, estudar, projetar e avaliar as práticas pedagógico - musicais referenciadas em um determinado modelo educacional, que obtêm, a partir da criatividade do professor, experiências criativas de aprendizagem.

O estudo propõe o desenvolvimento de estratégias criativas de ensino musical que partem do jogo como mediador, das experiências sensoriais como abordagem da experiência estética, da investigação e construção sonora com base no erro do aluno como reflexão e avaliação sobre o tema. ação; interrogando de um modo particular as práticas de educação musical.

Palavras-chave: Criatividade. Educação Musical. Educação Inicial. Práticas de Ensino. Estratégias de Ensino Criativo.

\section{Introducción}

La centralidad de este artículo, se expone en relación al estudio de prácticas creativas de enseñanza en Educación Musical, desde la reflexión acerca del planeamiento particular en el cual se desarrolla la misma, donde los obstáculos más frecuentemente observados se presentan como el desfasaje entre objetivos manifiestos (aquellos que se concretan) y objetivos deseados (aquellos que anteceden la práctica); y que se corresponden con investigaciones previas (Minist. Educ y C. Sta. Fe, 2004) en torno a evaluar el impacto de los documentos curriculares en cada nivel educativo; y su detección en relación al abordaje de los contenidos de expresión creativa dentro de los mismos.

Se postuló desde nuestra hipótesis inicial, que una manera de superar aquel desfasaje manifiesto entre la conceptualización y reflexión sobre un objeto disciplinar y su correspondiente experticia en el hacer creativo musical, sería en primer lugar: desde los aportes de la "Educación Experiencial" (construidos en dicha investigación y aplicados al objeto de estudio desde la definición y centralidad relacional en torno a las experiencias 
estéticas de Dewey (1934), los aportes del ciclo de Aprendizaje Experiencial - Reflexivo de Kolb (1984). En segundo lugar: desde los estudios y marco teórico en relación al enfoque experiencial y su inclusión dentro currículum (CAMILLONI, 2013), luego transferidos a la educación Inicial en el diseño de "secuencias didácticas flexibles" (GIGLIO, 2013). Reconociendo en tercer lugar: los aportes de la Psicología de la creatividad y los aportes de la Didáctica General al campo disciplinar de la Educación Musical.

Se consideraron los aportes de la Educación Experiencial entendida como estrategia de enseñanza con enfoque holístico dentro de cada currículo en particular que permite concebir el aprendizaje como resultado de una experiencia particular, relacionando el aprendizaje académico con la vida real, logrando en el proceso reflexivo la construcción de significado de dichas vivencias.

Por otro lado, se analizó la construcción de sentido en torno al conocimiento musical de las clases de nivel inicial como producto de estrategias de enseñanza diseñadas con enfoque articulador, que se efectivizan a partir del diseño de "Secuencias Didácticas Flexibles"ii (GIGLIO, 2013). A partir de las mismas, las actividades ideadas con determinados objetivos pedagógicos y medios, podían aplicarse durante un tiempo para lograrlos, de manera de posibilitar formularlas, adaptarlas, reformularlas y/o cambiarlas, y modificarlas según las diversas circunstancias (pp.77 - 86).

Ya que son las habilidades de razonamiento crítico y creativo aquellas que posibilitan aprender desde la experiencia, centrarse en la estimulación musical de la creatividad habilita miradas diversas. Entre ellas, el campo de la creatividad del docente, quien dispone de diversas secuencias posibles de enseñanza, constituyó una valiosa perspectiva de estudio en las clases de música del Nivel Inicial como puente para promover aquellos aprendizajes deseados.

\section{Educación Artística y creatividad en los primeros años de enseñanza general}

El siglo XXI encuentra a la Educación Artística en el centro de un entramado de ideas que la conducen al cuestionamiento, revisión y reconsideración de sus enfoques curriculares y metodológicos. 


\section{CREATIVIDAD EN LAS PRÁCTICAS DOCENTES DEL NIVEL INICIAL. UN ESTUDIO DESDE LA EDUCACIÓN MUSICAL}

En estos contextos tan complejos, cobra relevancia la idea de una educación integral que atienda diversas habilidades en los alumnos, y que posibilite el desarrollo de los niños desde edades tempranas atendiendo sus formas de aprehender y conocer el mundo. En dichas maneras de ser parte del mundo que los rodea, tanto docentes como familias, están atravesados por las circunstancias de la cultura contemporánea donde la educación (particularmente en el presente estudio en los primeros años de contactos con el arte musical), requiere de "docentes sensibles ante el mundo del arte, críticos -sobre todo auto-críticos-, y selectivos en relación a todo aquello que se vincule con el ambiente y las prácticas de aula" (IVALDI, 2014, p.p. 21-23). Esto implica contra-restar los estereotipos presentes en el entorno de los lenguajes artísticos, atendiendo así la preocupación actual de falta de flexibilidad y de creatividad ante propósitos determinados.

Desde estudios generales sobre creatividad, Guilford (1971) analiza situaciones que motivan o bloquean a los individuos para emprender una producción creativa, y propone responder a dichos interrogantes desde el foco de estudio en torno a la enseñanza. Torrance profundiza en investigaciones en los entornos escolares generales (1970 - 1977), analizando e infiriendo características de la capacidad creativa y su desarrollo. Csikszentmihalyi (1998) desde las contribuciones acerca del proceso creativo, avanza demostrando que los actos de naturaleza creativa son una conciliación entre el pensamiento convergente y divergente, y expone un enfoque que parece destinado a cambiar las ideas que se tienen sobre el tema dentro del campo de la educación. Para el autor antes mencionado, el criterio de fluidez se torna imprescindible de analizar en la búsqueda de nuevas vivencias y conocimientos que desarrollan y sostienen la curiosidad como habilitante de la originalidad (propia del pensamiento creativo).

En la misma línea de estudio, se considera a la creatividad como parte de un proceso, asociada con una conducta humana compleja (RUÍZ GUTIÉRREZ, 2010) y que es entendida holísticamente como parte de un todo interdependiente que supone el involucramiento de condiciones de una persona a través de articulados sucesos, etapas, niveles o hechos, que se despliegan y concretan para desarrollar la conducta creativa. Así mismo inscriben el concepto dentro de la categoría de: ambiente propicio (LÓPEZ PÉREZ, 1999), situado y contextualizado como parte de un fenómeno sistémico, más que individual (CSIKSZENTMIHALYI, 1998). En relación al proceso creativo y la determinación de sus etapas, Dewey en 1910 expuso cinco niveles del proceso creativo: el encuentro con una 
dificultad, tomando conciencia de que existe; la localización y precisión de la misma; el planteamiento de una posible solución o posibles soluciones; el desarrollo lógico de las consecuencias derivadas; y por último las observaciones y procedimientos experimentales que nos llevan a la aceptación o rechazo de la solución hipotética (p.72).

En torno a su definición y etapas o fases, se incluyen conceptualizaciones referidas específicamente a la educación, como la de Torrance (1976), desde donde se expresa la necesidad de comunicar los resultados a partir del proceso estudiado:

Creatividad es el proceso de ser sensible a los problemas, a las deficiencias, a las lagunas del conocimiento, a los elementos pasados por alto, a las faltas de armonía, etc.; de reunir una información válida; de definir las dificultades e identificar el elemento no válido; de buscar soluciones; de hacer suposiciones o formular hipótesis sobre las deficiencias; de examinar y comprobar dichas hipótesis y modificarlas si es preciso, perfeccionándolas y finalmente comunicar los resultados. (RUIZ GUTIÉRREZ, 2010, p.32)

Posteriormente, López Pérez (1999, p. 18) reúne algunas características en común no excluyentes ni únicas que consideran:

La creatividad como una actitud o una capacidad, que ubica el concepto en la categoría de persona (...) haciendo mención a la formación de combinaciones, relaciones o reestructuraciones, se incorpora la categoría de proceso (...) donde la creatividad debe manifestarse en algún resultado, ya sea material, concreto o intangible, con las características de lo original y relevante, lo que refiere a la categoría de producto.

En síntesis, es la creatividad - considerada dentro del ámbito de la educación artística - la que permite a las personas (como parte de una comunidad y sociedad) adaptarse a lo nuevo que acontece y transformar su realidad; mediante la imaginación e iniciativa creadora como parte del desarrollo social y cultural de los pueblos.

\section{Creatividad en los documentos curriculares de la Educación Inicial}

Desde los documentos curriculares vigentes en la Educación Inicial de la ciudad de Santa Fe (Ley de Educación Nacional No 26206) y Núcleos de Aprendizajes Prioritarios (NAP de la Educación Inicial -2004), se explicitan saberes que como sociedad se consideran 


\section{CREATIVIDAD EN LAS PRÁCTICAS DOCENTES DEL NIVEL INICIAL. UN ESTUDIO DESDE LA EDUCACIÓN MUSICAL}

claves, relevantes y significativos, con la finalidad de generar igualdad de oportunidades para todos los niños y niñas de la Argentina.

Dentro de la mención de dichos saberes culturales, sociales, y disciplinares se infieren diferentes situaciones de enseñanza que posibilitan promover variados modos de conocimiento musical en las salas de 5 años, perteneciente al primer nivel de la educación obligatoria. Se incluye así, la percepción y búsqueda de posibilidades expresivas, la producción y exploración del lenguaje musical, y el reconocimiento de manifestaciones artísticas del contexto cultural (CFE - NAP, 2011, p. 20). Estos núcleos de aprendizajes mencionados dentro de la Educación Inicial, interpelan las prácticas actuales de la enseñanza musical en el nivel, generando un marco de estudio en relación a las situaciones que las habilitan o posibilitan.

Con la intención explícita de recuperar saberes previos que entrelacen "modos personales de ser, hacer, pensar y sentir" (NAP 2011, p. 14) se expresa la importancia de pensar, diseñar, y ofrecer condiciones y ayuda pedagógica adecuada para brindar por medio de la actividad creadora experiencias de aprendizaje significativo, producto de la interacción del mundo interno y externo de cada persona.

\section{Creatividad en la enseñanza de la Música}

Circunscribiendo el estudio acerca del proceso creativo en la Educación Musical Argentina, se reconocen valiosos aportes, producto de investigaciones y experiencias al respecto. Frega $(1980 ; 2005 ; 2007 ; 2014 ; 2015)$ exploró diversos recortes y situaciones problemáticas que posibilitaron establecer los marcos adecuados para estimular la creatividad en procesos de educación musical dentro del entorno escolar general. Describe en sus investigaciones las disposiciones humanas naturales bajo cuatro aspectos a considerar en las experiencias: creador, intérprete, auditor y crítico (capacidades latentes en cada persona). Malbrán (2010) analizó diferentes atributos de la creatividad del docente que pueden considerarse como necesarios para el desarrollo posterior en los alumnos, entre ellos: la originalidad, la curiosidad, la resistencia, el carácter lúdico, la autoconciencia, la perseverancia, y la libertad; considerados como rasgos peculiares que se destacan en el comportamiento pedagógico creativo, y que "se convierten en metas trascendentes y deseables en los propios maestros” (p. 85). Espinosa, reconoció la creatividad como aquella facultad de la persona al permitirle resolver problemas de manera innovadora, considerando 
que: "para ser creativos hay que ser libres (...) y esa libertad también hay que conducirla con técnicas y estrategias para que esa nueva idea pueda ser aplicable" (2013, p. 10). Gainza, recuperó y expuso la importancia de estimular la participación de los estudiantes para explicar y verbalizar todo lo relacionado con la actitud de producción creativa, desde la autovaloración de cómo se le ocurrieron las ideas, cómo procedió, qué siente, qué busca, etc. destacando en tales conductas "el uso de los materiales, como de las experiencias musicales" (2002, p. 52).

Por lo expresado hasta aquí, se reconoce y analiza que existen diversos modelos de aprendizaje en relación a estudios del cómo se aprende creativamente; y desde esta investigación se persiguió el propósito de estudiar prácticas docentes creativas observables, identificables y evaluables en las clases de música de salas de 5 años; para inferir desde allí, estrategias de enseñanza creativas en la Educación Musical del Nivel Inicial.

La creatividad dentro de la didáctica de la educación musical posibilita analizarse desde diversas secuencias posibles de enseñanza a considerarse: analizando, indagando, observando y describiendo estrategias y situaciones que impulsen a crear nuevas respuestas, combinando nuevas ideas sonoras, saliéndose de las rutinas, cuestionando supuestos o estereotipos, tolerando ambigüedades o variedad de respuestas musicales, brindando andamiajes o soportes sonoros sobre los cuales se construye conocimiento, entre otros; y desde los cuáles se posibilita repensar las miradas creativas de los docentes como "puntapié inicial", de un cambio educativo real y posible.

\section{Construcción didáctica de la enseñanza musical creativa, en la Educación Inicial}

La posibilidad de propiciar desde las prácticas docentes el "hacer en música" (incluyendo la acción que ocurre dentro de la ejecución: en procedimientos, percepción y/o reflexión; como fuera del tiempo de ejecución en representaciones más estáticas o declarativas, presentaciones escritas o habladas y en el caso de alumnos del nivel inicial en el uso de metáforas, de comparaciones o de grafías de notación analógica), articula las habilidades que se regulan mutuamente en constante interacción, a modo de ciclo en espiral. Desde ese intercambio espiralado se posibilitarán posteriores exploraciones y creaciones sonoras $^{\mathrm{iii}}$, favoreciendo en la adultez las oportunidades de expresiones creativas. 


\section{CREATIVIDAD EN LAS PRÁCTICAS DOCENTES DEL NIVEL INICIAL. UN ESTUDIO DESDE LA EDUCACIÓN MUSICAL}

La percepción musical, la ejecución y la producción son aspectos posibles de enseñar y aprender en la educación Inicial ${ }^{\mathrm{iv}}$; y por ello, favorecer las experiencias relacionadas a estos modos de conocimiento musical desde la escolaridad general y desde las estrategias creativas del docente (en nuestro estudio en la enseñanza de música en sala de 5 años), posibilita una influencia significativa en el desarrollo musical posterior del alumno.

Swanwick (1991) analiza los primeros signos de expresión musical con el inicio de las canciones infantiles, y que ya a los cinco años comienzan a producir composiciones significativas posibles de ser estimuladas y potenciadas. La misma autora, y en colaboración con Tillman (1986) a través del análisis de las composiciones musicales de niños de 3 a 11 años, proponen un modelo en espiral de desarrollo musical en el que se describen ocho modos evolutivos comprometidos en el desarrollo musical (sensorial, manipulativo, personal, vernáculo, especulativo, idiomático, simbólico y sistemático). Debido a la etapa que nos ocupa es necesario centrarnos en las tres primeras, pero en directa incidencia con las posteriores. Desde allí, no sólo se establecen categorías que el docente debe conocer para desarrollar desde su construcción didáctica, sino que esto nos posibilita entender como la creatividad potencia y desarrolla "la imitación inicial"v que parece dominar en estos primeros años, hacia etapas posteriores de formas de expresiones personales, vernáculas socialmente compartidas.

\section{La Educación Experiencial en el estudio de prácticas docentes de la Educación Inicial}

Cuestionándonos inicialmente ¿cómo orientar la estimulación musical de la creatividad en el nivel inicial, de manera didáctica y sistemática?, se estudiaron una serie de actividades en las que a partir de esa conexión con la práctica musical, se solicitaba a los alumnos que pongan en juego habilidades y conocimientos que poseían (o que eran facilitados por el docente) a prueba en una situación auténtica, y que luego por medio de la reflexión sobre la acción (acompañada y mediada por el docente) se evaluaban sus resultados para abordar nuevos desafíos o problemas posteriores; generando consecuentemente, nuevas propuestas de enseñanza.

La consideración de la educación experiencial dentro el currículum de nivel inicial (bajo la cual subyace el modelo de aprendizaje experiencial [Kolb, 1984], el cuál describe la adquisición de conocimientos cuando el alumno observa y reflexiona sobre una experiencia 


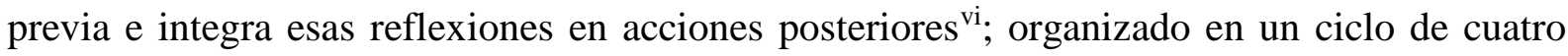
etapas sucesivas: - "Experiencia concreta" (EC) - "Observación Reflexiva" (OR) "Conceptualización Abstracta" (CA) - "Experimentación Activa" - EA), posibilitó entender que si el aprendizaje es favorecido desde la observación y la reflexión que se da sobre una experiencia previa, esto posibilita guiar acciones posteriores. Su consideración desde las estrategias musicales creativas del docente, es de vital influencia en el impacto sobre la enseñanza musical; en relación a la cantidad y calidad de las experiencias, y a la reconstrucción desde esa misma situación significativa ya adquirida como forma de retroalimentación.

Hace ya algunos años, Dewey expresó la existencia del arte en íntima relación con las experiencias como "aquella prueba viviente y concreta que el hombre es capaz de restaurar conscientemente, en el plano de la significación, la unión de los sentidos, necesidades, impulsos y acciones características de la criatura viviente" (1934 [1949], p. 25). Preocupado por la educación, y ante la posibilidad de extraer sentido de las experiencias presentadas, se pronunció al respecto:

Nuestra responsabilidad es conservar, transmitir, rectificar expandir la herencia de valores que hemos recibido de modo tal que quienes nos sucedan puedan recibirla más sólida y más segura, más ampliamente accesible y más generosamente compartida de lo que la recibimos nosotros. (DEWEY, 1934; en JACKSON, 2015, p. 73)

La enseñanza del lenguaje musical del nivel inicial, convertido en experiencia directa desde los aportes de la educación experiencial (cantar, bailar, crear, escuchar) potencia y posibilita desde nuestro enfoque, diversas formas de operar y de "hacer" con los variados tipos de conocimiento (declarativo y procedimental, DOWLING, 1992); posibilitando desde la construcción didáctica de cada docente las diversas manera de conocer en música, que abarcan modos de percepción, producción y reflexión (DAVIDSON y SCRIPP, 1992).

Se posibilita entonces, que cada docente articule sus propuestas de enseñanza y el contenido musical, habilitando el despliegue de un estilo particular, y construcción didáctica creativa, permitiendo "un proceso por el cual una vivencia o un conjunto de vivencias se convierte en "experiencias y éstas en aprendizajes" (CAMILLONI, 2013, p. 15). Este 


\section{CREATIVIDAD EN LAS PRÁCTICAS DOCENTES DEL NIVEL INICIAL. UN ESTUDIO DESDE LA EDUCACIÓN MUSICAL}

enunciado nos interpela de manera creativa en las prácticas situadas en el primer ciclo de la educación obligatoria, y nos moviliza a estudiarlas, recuperarlas y reflexionarlas.

\section{Aportes desde el diseño de Secuencias Didácticas Flexibles, para estudiar la enseñanza creativa de la música en el Nivel Inicial}

Desde las diversas vivencias favorecidas en clases de música, las experiencias sonoras enfocadas sistemáticamente en la educación inicial deben asegurar el "desarrollo estético, perceptivo y expresivo en un contexto de igualdad de oportunidades" (D. G. C. y E., 2007). Se destacan entonces, una serie de estudios de Giglio, vinculados a su tesis de doctorado (2010) e investigaciones posteriores desarrolladas entre Suiza, Argentina y Brasil, donde releva prácticas de enseñanzas innovadoras y creativas en el contexto de la formación docente que deben considerarse para desarrollar pautas de mejora en la educación musical. El autor estudia cinco momentos o dimensiones de las prácticas que apuntan a la innovación pedagógica centrados en la creatividad como el propósito del cambio. Entre ellas se destacan: la intención o propósito de cambio; la reacción de modificación con la posibilidad de predecir las prácticas creativas; la acción propiamente creativa desde el desarrollo de las funciones y competencias; la interacción social entre los individuos y las asociaciones de formación; y finalmente la adaptación y ajuste de prácticas o nuevos recursos tecnológicos para fines educativos creativos. Si bien estas dimensiones se estudiaron dentro de la formación docente a futuros maestros de música, se consideraron dentro de nuestro estudio como indicadores observables dentro de las características del docente creativo.

Desde dicho aporte, se reflexionó sobre el papel central que se le asigna a la creatividad en el diseño de actividades que parten desde los documentos curriculares, pero que reconocen como variables fundamentales las intenciones de los docentes en las aportaciones didácticas, más que desde dichas actividades prescriptas. Es por ello, que la participación, la observación y las modificaciones de la actividad creativa son colocadas en el centro de las secuencias didácticas flexibles, y habilitaron en nuestra investigación el estudio del "clima de formación creativa" (CAMILLONI, 2017) que involucra en la práctica docente momentos de exploración, investigación sonora, y desarrollo de la expresión musical sensibilizadora.

\section{Algunos aportes desde el estudio de caso}


Partiendo de los cuestionamientos acerca de ¿qué estrategias didácticas observables, identificables y evaluables permiten la experiencia creativa con el hacer musical favoreciendo los diferentes modos de conocimiento musical (apreciación, producción y creacióncontextualización) en la Educación Inicial?; se estudiaron aquellas condiciones que afectan el pensamiento creativo investigando que aspectos de las prácticas docentes en la enseñanza musical favorecen los procesos de elaboración y transformación de la información, para posibilitar al alumno la utilización del saber en otras conexiones y adaptaciones ingeniosas de nuevos usos.

El criterio para seleccionar los docentes de música $(n=3)$ contemplaron los prerequisitos de: formación académica de grado (universitario), carrera docente realizada en nivel universitario (Profesorado de música), pertenencia institucional (5 años al menos) y antigüedad en el cargo como docente titular en Jardines independizados de la educación Primaria. La recolección de datos se realizó por medio del análisis que se obtuvo desde la lectura de planificaciones y corpus documentales, las entrevistas estructuradas y semi estructuradas y las observaciones de clases a estos tres docentes de música del nivel inicial durante un mes de clases; para finalmente construir conocimiento didáctico acerca de las prácticas de enseñanza musical que expongan el desarrollo de la creatividad del docente, como estímulo para el aprendizaje musical.

Los datos del trabajo de campo se sometieron a un "análisis interpretativo" (ACHILLI, 2005, p. 40), desde donde la información dentro del proceso recursivo fue sometida a análisis crítico, contrastaciones, y triangulaciones; cuestión que posibilitó, la búsqueda de nexos conceptuales que construyeron y argumentaron el objeto de estudio, y así permitieron comprender los significados que producen los sujetos en dicho contexto estudiado. Así mismo, se establecieron comparaciones desde lo que dicen los docentes que hacen cuando realizan la planificación de sus propuestas de clase, y lo que sucede en las prácticas concretas de enseñanza (EDELSTEIN y CORIA, 1995).

Se utilizó para el análisis de datos, una herramienta para evaluar la actividad creativa en música denominada SCAMPER (FREGA - GARCÍA THIEME, 2005 - acrónimo de las voces inglesas «substitute, combine, adapt, modify: magnify or minify, put to other uses, 


\section{CREATIVIDAD EN LAS PRÁCTICAS DOCENTES DEL NIVEL INICIAL. UN ESTUDIO DESDE LA EDUCACIÓN MUSICAL}

eliminate, reverse», en castellano: sustituir, combinar, adaptar, modificar, magnificar o minimizar, poner en otros usos, eliminar, poner al revés). Desde dicha herramienta, se dispuso de descriptores objetivos y prácticos para estudiar las prácticas docentes en la conducción de los procesos creativos musicales. Desde allí, se cotejaron las conductas observables o identificadas, con la lectura de planificaciones y posteriormente con las inferencias desde las entrevistas y clases de los tres docentes. Se consideraron en su interpretación los cuatro criterios de estudios sobre creatividad de Guilford (1967), en relación a:

1)- flexibilidad de ideas (habilidad para reestructurar ideas y cambiar el flujo de una línea de pensamiento a otra, clasificables en categorías diferentes);

2)- fluidez de ideas (cantidad de ideas, respuestas o soluciones ante un problema concreto);

3)- originalidad de ideas (implica algo que sea distinto, soluciones infrecuentes, que no tiene precedentes);

4)- elaboración de ideas: capacidad para completar, llevar a cabo y/o concretar la respuesta.

Se infirió posteriormente a este proceso (desde los audios de entrevistas, los videos de observaciones de clases y los registros documentales aportados), aquellas estrategias que son favorables a la enseñanza creativa musical; y se triangularon en esta instancia los aportes de la educación experiencial y estimulación musical de la creatividad en la elaboración de secuencias didácticas flexibles.

Desde lo analizado y comparando a nivel horizontal (estableciendo similitudes, contradicciones o coincidencias entre los tres docentes), podemos destacar algunas dimensiones que se infieren como constantes, y que remiten a aspectos creativos del docente de música, en relación al despliegue de: curiosidad y entusiasmo; iniciativa y respeto a la diversidad; flexibilidad y fluidez; disponibilidad al cambio; originalidad en sus propuestas; lugar a lo imprevisto; juicio y espíritu crítico; sensibilidad y empatía; formación musical continua y originalidad en sus propuestas. 
Se postuló finalmente desde el presente estudio la articulación de los aspectos creativos mediante el diseño de la Propuesta de Secuencia Cíclica de Prácticas Creativas en Educadores Musicales, que permite una integración holística de lo observado y estudiado en la clase de música de la educación inicial. Dentro de esta propuesta se consideraron aspectos centrales, que incluyen dimensiones a considerase desde una revisión crítica, comprendiendo: EL JUEGO en las experiencias musicales y sensoriales - la construcción de conocimiento musical desde el ERROR del alumno - la INDAGACIÓN como estrategia creativa del docente - el uso del CUERPO como forma de acercamiento particular de cada persona a la

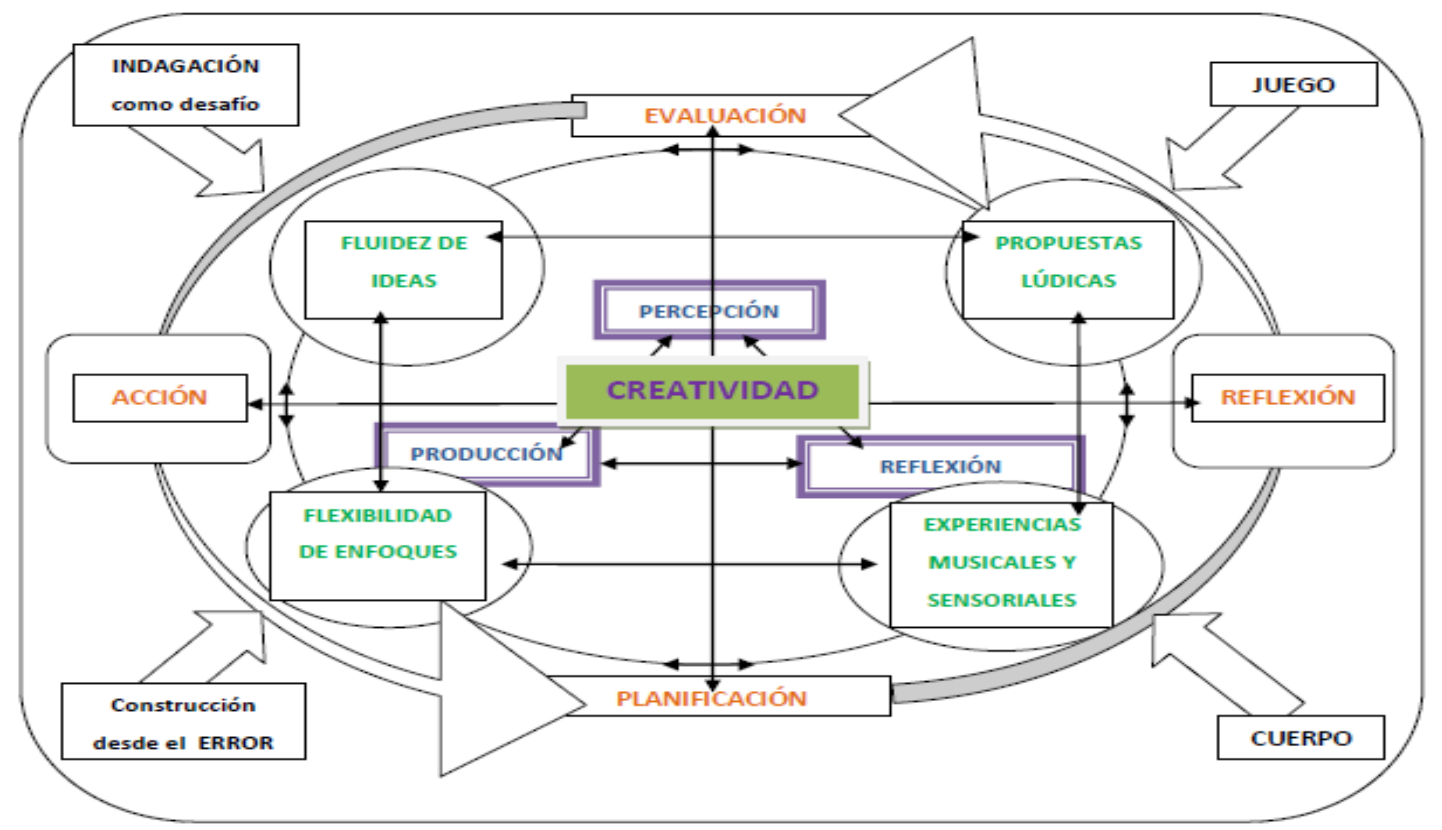

música - la PLANIFICACIÓN como construcción reflexionada y contextualizada "en" y "para" la creatividad - y la EVALUACIÓN entendida como punto de inflexión en las prácticas creativas de los docente.

Cuadro1. Propuesta de Secuencia Cíclica de prácticas creativas en Educadores Musicales, (ZILLI, 2017) 


\section{CREATIVIDAD EN LAS PRÁCTICAS DOCENTES DEL NIVEL INICIAL. UN ESTUDIO DESDE LA EDUCACIÓN MUSICAL}

\section{Conclusiones: Propuesta de Secuencia Cíclica de Prácticas Creativas en Educadores Musicales}

En contextos educativos actuales tan complejos, cobró relevancia la idea de una educación integral que atienda diversas habilidades en los niños, y que posibilite el desarrollo multidimensional de los mismos desde edades tempranas atendiendo sus diversas formas de aprehender y conocer el mundo de manera creativa. En directa vinculación, el estudio sobre el desarrollo de estrategias creativas de enseñanza cobró relevancia como estímulo para la construcción de conocimiento disciplinar musical, desde diversas instancias que se reconocieron como posibilitadoras de una Educación en Creatividad.

Desde los aportes de dicha investigación se habilitan caminos posibles a recorrer y retomar; susceptibles de abordarse desde cualquier aspecto allí mencionado, y que no requiere de un tratamiento sucesivo fijo, ni del recorrido lineal de dicha secuencia. Se consideran en dichas conclusiones (cuadro 1), el despliegue de las prácticas creativas que el docente realiza cuando: concibe el juego como estrategia articuladora que favorece el desarrollo de la imaginación, de la curiosidad, y de la diversidad de experiencias lúdicas; utiliza el cuerpo como vehículo de apropiación sensorial y musical, que habilita al alumno para favorecer el aprendizaje comprensivo de la música; incluye la posibilidad de abordar la enseñanza a partir del error habilitando multiplicidad de caminos y soluciones creativas ante estos problemas particulares que permiten construir conocimiento posterior. Por otro lado, cuando propone la indagación como estrategia entendida desde la posibilidad de acción, que tiende a la actitud crítica y revisionista en la apertura ante diversas experiencias musicales desde pensamiento creativo. Y finalmente cuando reflexiona sobre la planificación y los modos de evaluar en música (que necesitan repensarse desde un currículum) partiendo de las necesidades del contexto socio cultural, recuperando sus inquietudes y potenciando la creatividad en el aula; además cuando sistematizan algunas estrategias dentro del diseño de clases (unidades, proyectos, fichas para los alumnos, etc.) que configuran nuevas maneras de diseñar prácticas de enseñanza musical en la Educación Inicial.

Se confirma desde este estudio, el desarrollo creativo de los docentes como potenciadores del pensamiento creativo en los alumnos. Caracterizado por una permanente reflexión y revisión de las estrategias, se permite volver a las acciones con una mirada crítica, posibilitando el cambio en todos los actores de la escena educativa y habilitando a la vez, la 
búsqueda de los estados intencionales que hay detrás de las acciones dando lugar a la expresión musical, la creatividad, y el conocimiento compartido.

\section{Referencias}

ACHILLI, E. Investigar en Antropología social. Los desafíos de transmitir un oficio. Rosario: Ed. Laborde Libros, 2005.

CAMILLONI, A. La inclusión de la educación experiencial en el currículo universitario. En: Menéndez y otros, Integración, docencia y extensión. Otra forma de enseñar y de aprender (pp. 11-21). Santa Fe: UNL, 2013.

CAMILLONI, A. Una enseñanza orientada al desarrollo de la creatividad. Conferencia 7/04/2017. Paraninfo UNL. Disponible en LITUS EDUCA: https://www.youtube.com/watch?v=wsOmCulgwCE (consulta: mayo 2017), 2017.

C.F.E. Ley de Educación Nacional No 26.206, 2006.

C.F.C.yE. y Ministerio de Educación de la Nación Núcleos de Aprendizajes Prioritarios (NAP) Educación Inicial. 2a edición: octubre de 2011. Buenos Aires, 2004.

CSIKSZENTMIHALYI, M. Creatividad: Flujo y la psicología del descubrimiento y la invención. Nueva York, Nueva York: HarperCollins, 1996.

DAVIDSON, L. Y SCRIPP. L. Surveying the coordinates of cognitive skills in music. En Colwell, R. (ed) Handbook of research on music teaching and learning. Chapter 25: 392413. New York, USA: Shimer Books. Traducido por Carmen Fernández, 1992.

DELALANDE, F. El rol de los dispositivos en una pedagogía de la creación musical. Inst. Nac. del Audiovisual - Grupo de Investigaciones Musicales. París, Francia Traducción: María Guadalupe Segalerba. Consulta mayo 2014, recuperado de: file:///D:/Mis\%20Cosas/Descargas/DELALANDE+(+el+rol+de+los+dispositivos), 2009.

DEWEY, J. El Arte como experiencia. Nueva York, Minton, Balch \& Co. Edición en español (1949) México: Fondo de Cultura Económic, 1934.

DOWLING, J. Conocimiento Procedimental y conocimiento declarativo en educación y cognición musical. Traducción: I. Mártinez. Bs. As.: Conservatorio Alberto Ginastera, 1993.

EDELSTEIN, G Y CORIA, A. Imágenes e imaginación. Iniciación a la docencia. Buenos Aires: Kapeluz, 1995. 


\section{CREATIVIDAD EN LAS PRÁCTICAS DOCENTES DEL NIVEL INICIAL. UN ESTUDIO DESDE LA EDUCACIÓN MUSICAL}

ESPINOSA, S. La creatividad como camino para la creación musical. Aprender haciendo, hacer jugando, jugar creando. En Artes integradas y educación, punto de interacción creativa: libro 3. Bs. As.: Ediciones UNLa - Universidad Nacional de Lanús, 2013.

FREGA, A. y VAUGHAN, M. Creatividad musical: Fundamentos y estrategias para su desarrollo. Buenos Aires: División Desarrollo Musical Casa América, 1980.

FREGA, A. Y GARCÍA THIEME, M. F. El S.C.A.M.P.E.R como herramienta de análisis: la flexibilidad en situaciones educativas de estimulación sistemática de la creatividad. Boletín de Investigación Educativo-Musical del CIEM, año 12, n. 35, p. 42-48, 2005.

FREGA, A. Educar en creatividad. Bs. As.: Academia Nacional de Educación, 2007.

Creatividad y Educación Musical. N¹3. pág 10 a 31. Madrid, 2009. Recuperado de: www.creatividadysociedad.net (fecha de consulta febrero 2014).

FREGA, A. Evaluar experiencias de creatividad musical en el aula: informe sobre una línea de investigación. Porto Alegre, Brasil: Revista da ABEM, v24, 13-24, 2010.

Creatividad en enseñanzas artísticas. Journal for Educators, Teachers and Trainers, Vol. 5 (1), pp. 6-8, 2014.

La alfabetización en creatividad. En: Barcia, Ratto (coords.) Reflexiones sobre la formación docente - Academia Nacional de Educación. Buenos Aires: Ed. Dunken, 2015.

GAINZA, V. Pedagogía Musical. Dos décadas de pensamiento y acción educativa. Bs.As.: Ed. Lumen, 2002.

GIGLIO, M. Colaboración creativa y musical entre alumnos y acciones de sus docentes en contexto escolar: síntesis de una investigación. Tese (Doutorado em

Educação). (Recuperado

de:

http://www.seer.ufu.br/index.php/emrevista/article/viewFile/14916/8412 Ensino

Em ReVista, v. 19, n. 1, jan./jun. 2012 (consulta: marzo 2015) Suiza: Universidad de Neuchâtel, 2010.

Five dimensions to study teacher education change for improving musical creative learning. Journal for Educators, Teachers and Trainers, Vol.

5 (1), pp. 80-89, 2013.

Cuando la colaboración creativa cambia la forma de enseñar: desarrollo y observaciones de una propuesta pedagógica desde la educación musical. Santander, España: Ed. Universidad de Cantabria, 2013.

GUILFORD, J.P. Intelligence, creativity, and their Educational Implications. Ed. Knapp, 1968.

GUILFORD, J. P. y otros. Teachers and the learning process En español: Creatividad y Educación (1978). Buenos Aires: Ed. Paidós, 1971. 
IVALDI, E. Educación, arte y creatividad en las infancias del siglo XXI. En SARLÉ, P.; IVALDI, E.; HERNANDEZ, L. Arte, Educación y Primeras Infancias. España, Organización de Estados Iberoamericanos para la Educación, la Ciencia y la Cultura, 2014.

JACKSON, P. ¿Qué es la educación? Trad. Alicia Brixio. Bs.As.: Ed. Paidós, 2015.

KOLB, D. Experiential Learning. Englewood Cliffs, N.J.: Prentice-Hall, 1984.

LÓPEZ PÉREZ, R. Prontuario de la creatividad. Chile: Universidad Educares, Bravo y Allende, 1999.

MALBRÁN, S. La creatividad de los maestros y la educación musical. Rev. Creatividad y Sociedad nº 13. Pág. 80 a 105. Madrid, Recuperado de www.creatividadysociedad.net, 2009. M. E. y C., Ministerio de Educación de la Provincia de Santa Fe Investigación cooperativa: "Evaluando junto a los docentes el impacto de los diseños curriculares jurisdiccionales en las prácticas educativas" - Programa de Investigación educativa. Santa Fe: Imprenta Oficial, 2004.

RUIZ GUTIÉRREZ, S. Práctica educativa y creatividad en educación infantil. Tesis de doctorado. Universidad de Málaga. Recuperado (enero 2016): https://riuma.uma.es//xmlui/handle/10630/4618, 2010.

SWANWICK, K. Música, pensamiento y educación. Madrid: Morata, 1991.

SWANWICK, K. Y TILlMAN, J. The Sequence of Musical Development: A Study of Children's Composition. British Journal of Music Education - Volume 3 (3), p. 331. 1986.

TORRANCE, E. P. Desarrollo de la creatividad del alumno. Trad. Rodolfo Schwarz. Buenos Aires: Librería del Colegio, 1970.

TORRANCE, E. P. Educación y capacidad creadora. Madrid, España: Ed. Marova, 1977.

\section{Notas}

\footnotetext{
${ }^{\text {i }}$ Dicha investigación corresponde a la tesis de Maestría en Didácticas Específicas (FHUC - UNL), presentada y defendida en diciembre 2017.

ii Se definen como "un ordenamiento temporal más general de actividades en el que el docente se preocupa por llevar a cabo ciertas acciones educativas para resolver permanentemente situaciones y problemas concretos de enseñanza y aprendizaje" (GIGLIO, 2013, p.77); identificando el contenido curricular a enseñar (objeto de saber) y transformándolo en válido para enseñar (transposición didáctica). Las denomina como flexibles, ya que el docente las "adapta a las circunstancias sin dudar en modificar, si es necesario, lo que ha previsto" (idem, p.83).
} 

ESTUDIO DESDE LA EDUCACIÓN MUSICAL

\footnotetext{
iii Delalande especifica desde la pedagogía musical la posibilidad de ampliar el campo de la música con diversos géneros y estilos, posponiendo la enseñanza de notación tradicional para otros ciclos posteriores o instituciones especializadas para tal fin, y lo principal "otorgando el lugar central a una actividad de producción que apele a la creatividad" (2009, p. 1).

iv Sloboda (1985) establece una diferenciación entre "aculturación musical", como el desarrollo musical espontáneo, sin ningún esfuerzo ni dirección; y por otro lado el "entrenamiento" como esfuerzos autoconscientes dirigidos para mejorar destrezas musicales específicas y que se inician en la escolaridad general.

${ }^{v}$ En referencia a estas primeras etapas, Swanwick señala que "la imitación no es mera copia, sino que incluye la afinidad, la empatía, la identificación, el interés viéndonos como otra cosa u otra persona (...) es tan inevitable como el gusto por el dominio de los materiales, y no es contraria a la imaginación creativa" (1991, p. 52).

vi Si bien este modelo pedagógico que subyace desde el proyecto denominado "aprendizaje experiencial reflexivo" (KOLB, 1984) fue diseñado inicialmente en la Universidad de Hardvard (desde las problemáticas detectadas en el nivel Superior y centradas en torno a la enseñanza que favorece el "aprender a aprender" de los alumnos); podemos desde la presente investigación recuperar sus aportes para considerarlos en la enseñanza del Nivel Inicial, desarrollando habilidades de pensamiento creativo y crítico posterior.
}

\section{Sobre a autora}

Zilli, Lía Rosa. Profesora de Música egresada del Instituto Superior de Música (UNL). Magíster en Didácticas Específicas (FHUC - UNL). Actualmente es profesora del Instituto Superior de Música (UNL), e Institutos de Formación docente de la ciudad de Santa Fe (IS $\mathrm{N}^{\circ} 32$, IS $\mathrm{N}^{\circ} 8$, ISPI $\mathrm{N}^{\circ} 9105$ ) en los profesorados de Educación Inicial, Educación Primaria, y Educación Especial. Correo Electrónico: liarzilli@gmail.com 